Article

\title{
Variability of DNA Methylation within Schizophrenia Risk Loci across Subregions of Human Hippocampus
}

\author{
W. Brad Ruzicka ${ }^{1,2, *}$, Sivan Subburaju ${ }^{1,2}$ and Francine M. Benes ${ }^{1,2,3}$ \\ 1 Program in Structural and Molecular Neuroscience, McLean Hospital, Belmont, MA 02478, USA; \\ ssubburaju@mclean.harvard.edu (S.S.); fbenes@mclean.harvard.edu (F.M.B.) \\ 2 Department of Psychiatry, Harvard Medical School, Boston, MA 02478, USA \\ 3 Program in Neuroscience, Harvard Medical School, Boston, MA 02478, USA \\ * Correspondence: wruzicka@mclean.harvard.edu; Tel.: +1-617-855-3085
}

Academic Editor: Dennis R. Grayson

Received: 7 March 2017; Accepted: 11 May 2017; Published: 15 May 2017

\begin{abstract}
Identification of 108 genomic regions significantly associated with schizophrenia risk by the Psychiatric Genomics Consortium was a milestone for the field, and much work is now focused on determining the mechanism of risk associated with each locus. Within these regions, we investigated variability of DNA methylation, a low-level cellular phenotype closely linked to genotype, in two highly similar cellular populations sampled from the human hippocampus, to draw inferences about the elaboration of genotype to phenotype within these loci enriched for schizophrenia risk. DNA methylation was assessed with the Illumina HumanMethylation450 BeadArray in tissue laser-microdissected from the stratum oriens of subfield CA1 or CA2/3, regions having unique connectivity with intrinsic and extrinsic fiber systems within the hippocampus. Samples consisted of postmortem human hippocampus tissue from eight schizophrenia patients, eight bipolar disorder patients, and eight healthy control subjects. Within these genomic regions, we observed far greater difference in methylation patterns between circuit locations within subjects than in a single subregion between subjects across diagnostic groups, demonstrating the complexity of genotype to phenotype elaboration across the diverse circuitry of the human brain.
\end{abstract}

Keywords: DNA methylation; HumanMethylation450; schizophrenia; bipolar disorder; postmortem human brain; epigenetics; hippocampus

\section{Introduction}

Recent years have brought exciting advances in our understanding of the genetic underpinnings of major mental illnesses with the progress of next-generation sequencing technologies and bioinformatic methods for analysis of the complex datasets they produce. The identification of 108 genome-wide significant schizophrenia associated genomic regions by the Psychiatric Genomics Consortium (PGC) in 2014 brought robust results from the field's considerable investment into genome-wide association studies, although now with these regions in hand the quest to understand this debilitating disorder is of course still far from complete. These regions span more than 20 megabases of DNA (20877196 base pairs [1]), approximately two thirds the size of the entire human exome [2], and are estimated to confer only $3.4 \%$ of the variance in schizophrenia risk [3], leaving the bulk of disease liability still unexplained.

Schizophrenia is a neurodevelopmental disorder that likely involves molecular pathologies throughout the vastly complex system of the human brain, shaped by genetic and environmental influences spanning the time from early development throughout the lifespan [4]. Genotype is the foundation from which the multitude of phenotypes expressed in any single cell (the epigenome, 
transcriptome, spliceosome, proteome, and so on) are elaborated through interaction with environmental [5] and stochastic factors [6] that are not as yet fully understood. In the central nervous system, phenotypes of single neurons or glia interact at the levels of cell types, individual regions and larger networks of regions to produce the phenotype of the entire individual, from which a complex syndrome such as schizophrenia or bipolar disorder may be displayed. In the context of the daunting complexity presented by these and other neuropsychiatric disorders whose pathology lies within the brain, it is pertinent to consider what the significance of disease-associated single nucleotide polymorphisms (SNPs) identified in peripheral tissues such as blood may be.

It is likely that epigenetic mechanisms play an important role during brain development and throughout the life cycle in defining the ways in which an individual's genotype is expressed as unique molecular phenotypes within specific neuronal subtypes in subregions of the brain. With the goal of accessing this process occurring within the central nervous system, specific DNA methylation signatures have been suggested as biomarkers of disease in peripheral blood that may have utility in clinical diagnosis or treatment, but more work is needed before meaningful constructs emerge [7]. The utility of biomarkers of this type is complicated by the fact that DNA methylation patterns show more variance between separate tissues as well as subregions within the brain of a single individual than they do between a single subregion across multiple subjects. This has been reported for peripheral blood mononuclear cells as compared to multiple human brain regions $[8,9]$.

Many groups are now working to investigate the mechanisms conferring risk associated with each PGC region, and DNA methylation is one potential mechanism of risk receiving a great deal of attention. Methylation quantitative trait loci (meQTLs) are genomic regions where the DNA sequence influences methylation of a region or regions of DNA in cis or in trans [10], and this effect may be specific to distinct cell or tissue types and developmental time periods. Two important recent studies $[11,12]$ investigated postmortem human brain tissue across the lifespan to demonstrate that PGC regions are enriched for sites that are differentially methylated between pre- and post-natal life. Also, it was discovered that nearly $60 \%$ of PGC regions contain a significant meQTL, potentially contributing to those regions' mechanisms of risk. These studies analyzed samples of homogenized whole cortex, and changes in DNA methylation across development were found to be related to shifting proportions of different cellular populations within the brain [11]. Also, while the first study investigated only dorsolateral prefrontal cortex in adult brain, the second compared three regions (prefrontal cortex, striatum, and cerebellum) and found that while most fetal meQTLs were stable, there were meQTLs that showed specificity with regard to time and brain region [12].

In light of these recent findings, we sought to investigate the variability of DNA methylation patterns within the 108 PGC regions across closely related circuit locations within the human hippocampus, and how this differs within and between individuals and diagnostic groups. The work described herein is a focused secondary analysis of a previously reported investigation of DNA methylation patterns in two distinct but highly similar cell populations sampled from hippocampal subregions CA1 and CA2/3 [13]. These subregions were sampled from a cohort of 24 postmortem human hippocampus tissue samples obtained from eight schizophrenia (SZ) patients, eight bipolar disorder (BD) patients, and eight healthy control (CON) subjects. Laser microdissection was used to dissect tissue specifically from the stratum oriens, the outermost cellular layer of the hippocampal formation containing exclusively GABAergic interneurons as well as supportive non-neuronal cell types [14], from subfields CA2/3 and CA1, the locations of the second and third synapses within the trisynaptic pathway, respectively. DNA methylation was assessed in each of the 48 samples using the Illumina HumanMethylation450 BeadArray, and the analysis was restricted to the 5476 probes assessing cytosine residues found within the 108 PGC regions. Consistent with prior studies [8], our results demonstrate greater differences in methylation patterns between circuit locations within individuals than between single circuit locations across diagnostic groups. These data reinforce the difficulty of extrapolating genetic signals to phenotypic events across distinct tissues, and showcase one 
aspect of heterogeneity within the brain that cannot be appreciated through measurements performed in homogenized whole brain structures or in peripheral tissues.

\section{Materials and Methods}

\subsection{Postmortem Human Hippocampus Tissue Samples}

This work investigated a previously described [13] cohort containing postmortem human hippocampus tissue samples from 24 individuals, eight with schizophrenia, eight with bipolar disorder, and eight healthy controls, obtained from the Harvard Brain Tissue Resource Center (Table 1). Groups were matched for gender, age, postmortem interval, and tissue $\mathrm{pH}$. All cases were obtained through family referral, not through the office of a medical examiner, and cases with documented history of serious drug abuse were excluded.

On arrival to the center, the fresh brains were dissected and the hippocampus was removed and sectioned. Tissue blocks used in this study were taken from the level of the pulvinar thalami along the rostro-caudal axis of the hippocampus. Upon removal, tissue blocks were fixed in ice-cold $0.1 \%$ formalin in $0.1 \mathrm{M}$ phosphate buffer ( $\mathrm{pH} 7.2$ ) for $90 \mathrm{~min}$ before cryoprotection with $30 \%$ sucrose in phosphate buffer overnight and then stored at $-80^{\circ} \mathrm{C}$.

Table 1. Summary of demographic measures within the cohort.

\begin{tabular}{cccccc}
\hline Diagnosis & $\boldsymbol{n}$ & Gender & Age & PMI & pH \\
\hline CON & 8 & $4 \mathrm{M} / 4 \mathrm{~F}$ & $64.1 \pm 14.2$ & $22.4 \pm 5.7$ & $6.4 \pm 0.2$ \\
SZ & 8 & $4 \mathrm{M} / 4 \mathrm{~F}$ & $67.9 \pm 17.3$ & $26.4 \pm 10.3$ & $6.4 \pm 0.2$ \\
BD & 8 & $3 \mathrm{M} / 5 \mathrm{~F}$ & $68.3 \pm 11.7$ & $25.0 \pm 6.7$ & $6.3 \pm 0.2$ \\
$p$ & & 0.86 & 0.82 & 0.58 & 0.46 \\
\hline
\end{tabular}

Demographic variables averaged within each diagnostic subgroup of the cohort are listed, with $p$ values for each measure generated by ANOVA provided in the final row. Full details for individual subjects are available in a previous description of this cohort [13]. CON: control; SZ: schizophrenia; BD: bipolar disorder; PMI: postmortem interval.

\subsection{Sample Processing and DNA Methylation Measurement}

Tissue blocks were embedded in Optimal Cutting Temperature compound (Sakura Finetek, Torrance, CA, USA) and cut to $30 \mu \mathrm{m}$ at $20^{\circ} \mathrm{C}$ in a Microm HM560 cryostat. Sections were mounted on polyethylene terephthalate frame slides (Leica, Wetzlar, Germany), Nissl stained and dehydrated with ascending concentrations of ethanol. Sections were dried and stratum oriens tissue was dissected from CA1 and from CA2/3 using a Leica LMD6500 laser microdissection system. DNA was extracted from each sample using the QIAamp DNA Micro Kit (Qiagen, Hilden, Germany) and stored at $-80^{\circ} \mathrm{C}$.

$500 \mathrm{ng}$ of genomic DNA was bisulfite modified using the EZ DNA Methylation Kit (Zymo Research, Irvine, CA, USA) using modified parameters recommended by Illumina, and modified DNA was assessed using the Illumina HumanMethylation450 BeadArray (Illumina, San Diego, CA, USA) as per the manufacturer's protocol.

\subsection{Data Analysis}

Data was analyzed within $R$ version 3.3.2. (R Foundation, Vienna, Austria). Raw intensity idat files were read into the minfi Bioconductor package [15] and whole-array data was normalized by stratified quantile normalization. No samples were identified as outliers and all samples were included in the analysis. M values (logit transformed $\beta$ values which represent methylation ratios between 0 and 1 ) were extracted and then the dataset was restricted to the 5476 probes assessing cytosine residues within the original 128 genomic regions reported by the PGC [1]. Differentially methylated positions (DMPositions) were identified with false detection rate (FDR) $<0.05$ using linear regression with the DMRCate Bioconductor package's "myannotation" function [16] controlling for case (in comparisons across regions within individual subjects), age, and postmortem interval (PMI). Differentially 
methylated regions (DMRegions) were identified with Stouffer corrected $p$ value $<0.05$ with the "dmrcate" function within the DMRCate Bioconductor package, again with case (in comparisons across regions within individual subjects), age, and PMI included as covariates.

\section{Results}

\subsection{Differentially Methylated Positions}

The Illumina HumanMethylation450 BeadArray was used to investigate diagnosis associated changes in DNA methylation within the 108 PGC schizophrenia associated genomic regions in postmortem human hippocampus tissue from schizophrenia, bipolar disorder, and healthy control subjects. Investigation of the data for DMPositions identified only a single significant diagnosis associate difference among all four comparisons ( 5476 probes compared between SZ CA1 and CON CA1, SZ CA2/3 and CON CA2/3, BD CA1 and CON CA1, and BD CA2/3 and CON CA2/3). In the SZ CA2/3 vs. CON CA2/3 comparison, probe $\operatorname{cg} 07925823$ within the first exon of the SLC7A6 gene on chromosome 16 was significantly different between groups with FDR $=6.2 \times 10^{-3}$.

Assessment of differences between circuit locations, on the other hand, identified 497 DMPositions among all three comparisons (5476 probes compared between SZ CA1 and SZ CA2/3, BD CA1 and BD CA2/3, and CON CA1 and CON CA2/3) (Figure 1a, Table 2 and Supplementary Materials file), and 966 DMPositions between CA1 and CA2/3 by pooling all 24 cases without consideration of diagnostic categories (Figure $1 \mathrm{~b}$ and Supplementary Materials file). In Figure 1a, it is apparent that DMPositions within and between diagnostic groups cluster around certain genomic loci, generating the loosely columnar appearance of the plot. More than $50 \%$ of individual probes corresponding to circuit location DMPositions were shared between separate diagnostic subgroup comparisons, and $95 \%$ to $99 \%$ of individual DMPositions identified in circuit location comparisons within diagnostic subgroups were also identified as DMPositions in the pooled analysis (Table 2).

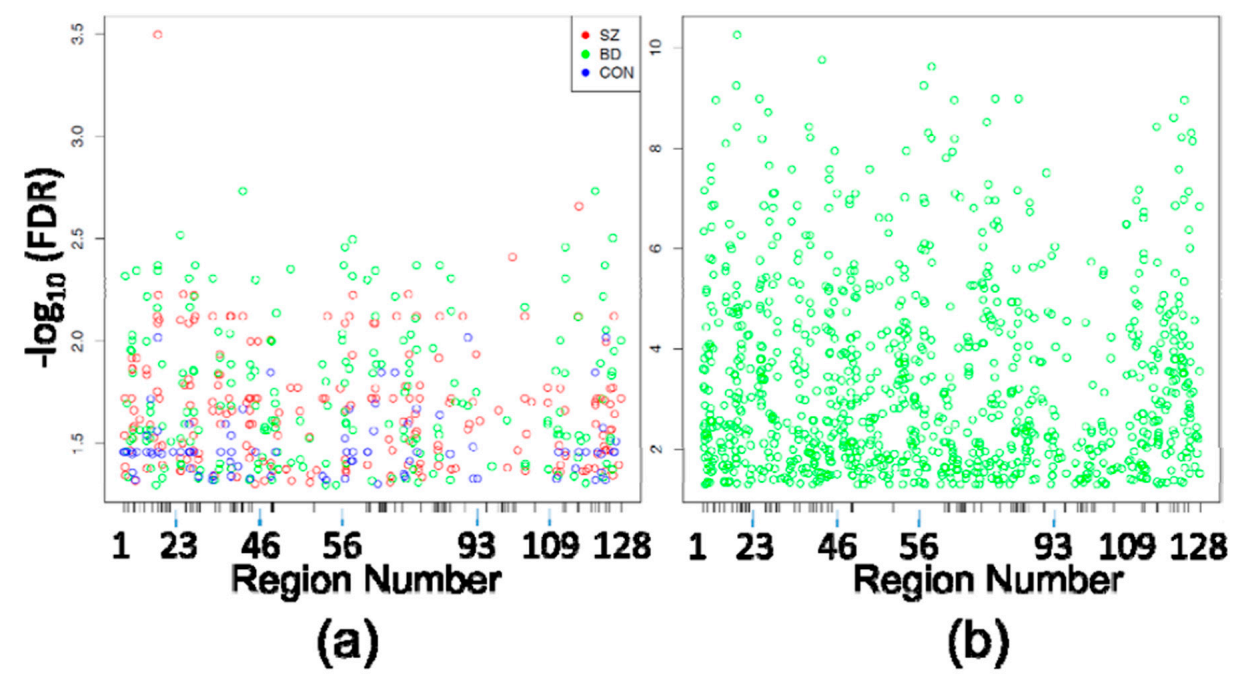

Figure 1. Differentially Methylated Positions (DMPositions) in CA1 vs. CA3. (a) Shown are the DMPositions identified within each eight-case diagnostic subgroup comparison (210 in SZ CA1 vs. SZ CA2/3, 201 in BD CA1 vs. BD CA2/3, 86 in CON CA1 vs. CON CA2/3) with genomic location within the space corresponding to the $5476 \mathrm{HM} 450$ probes assessing sites within the original 128 PGC regions (5476 probes within those regions ordered from chromosome 1 to chromosome $X, p$ to $q$ ) depicted on the $\mathrm{X}$ axis, and the $-\log 10$ (FDR) on the $\mathrm{Y}$ axis. $\mathrm{X}$-axis ticks mark the boundaries between PGC regions. As seen by the loose vertical columns, the DMPositions from separate diagnostic subgroups have a high degree of overlap, which is detailed in Table 2; (b) The 966 DMPositions identified in the pooled analysis of all 24 CA1 samples vs. all 24 CA2/3 samples are depicted within the same $X$ and Y axes as Figure 1a. 
Table 2. Distribution and overlap of differentially methylated positions among diagnosis and circuit location comparisons.

\begin{tabular}{cccc}
\hline Comparison & \# of DMPs & Mean Delta Beta & Mean FDR \\
\hline SZ CA1 vs. SZ CA2/3 & 210 & 0.064 & 0.025 \\
BD CA1 vs. BD CA2/3 & 201 & 0.063 & 0.023 \\
CON CA1 vs. CON CA2/3 & 86 & 0.075 & 0.034 \\
Pooled Cases CA1 vs. CA2/3 & 966 & 0.035 & 0.0087 \\
SZ CA2/3 vs. CON CA2/3 & 1 & 0.035 & NA \\
SZ CA1 vs. CON CA1 & 0 & NA & NA \\
BD CA2/3 vs. CON CA2/3 & 0 & NA & NA \\
BD CA1 vs. CON CA1 & 0 & \% of DMPs & \\
\hline Comparison & \# of DMPs & 53.23 & \\
\hline SZ vs. BD & 107 & 61.63 & \\
SZ vs. CON & 53 & 68.6 & \\
BD vs. CON & 59 & 53.49 & \\
All 3 Groups & 46 & 94.76 & \\
SZ vs. Pooled & 199 & 97.01 & \\
BD vs. Pooled & 195 & 98.83 & 100 \\
CON vs. Pooled & 85 & 46 & \\
All vs. Pooled & 46 & & \\
\hline
\end{tabular}

At the top are listed the number of DMPositions identified in each circuit location (i.e., SZ CA1 vs. SZ CA2/3) or diagnostic group (i.e., SZ CA1 vs. CON CA1) comparison, and the mean delta beta and mean FDR for all DMPs in that comparison. At the bottom is the overlap of specific CpG sites among the various comparisons, with \# of DMPs indicating the number of DMPositions that are common to the two listed comparisons (i.e., $\mathrm{SZ}$ vs. BD indicates there are 107 DMPositions common to both the SZ CA1 vs. SZ CA2/3 and the BD CA1 vs. BD CA2/3 comparisons). All three groups indicate there are 46 DMPositions common to all three within diagnostic subgroup comparisons. $\%$ of DMPs lists the percentage of DMPositions from the comparison with the smaller number of DMPositions that are common to both indicated comparisons (i.e., for SZ vs. BD 107 common DMPositions/201 BD CA1 vs. BD CA2/3 DMPositions = 53.23\%). DMP: differentially methylated position; FDR: false detection rate.

\subsection{Differentially Methylated Regions}

Investigation of DMRegions yielded similar results, with zero significant DMRegions detected in any of the four between-diagnosis comparisons described above, and multiple DMRegions between each circuit location within diagnostic subgroups. Figure 2a shows the 19 significant DMRegions between SZ CA1 and SZ CA2/3, 23 DMRegions between BD CA1 and BD CA2/3, and ten DMRegions between CON CA1 and CON CA2/3, and shown in Figure $2 b$ are the 103 significant DMRegions between CA1 and CA2/3 in all cases, pooled without consideration of diagnosis. As with DMPositions, there is a large amount of overlap of circuit location of DMRegions between the diagnostic subgroups (Table 3), with approximately half as many DMRegions observed in the control subjects as there were in each of the patient groups. 


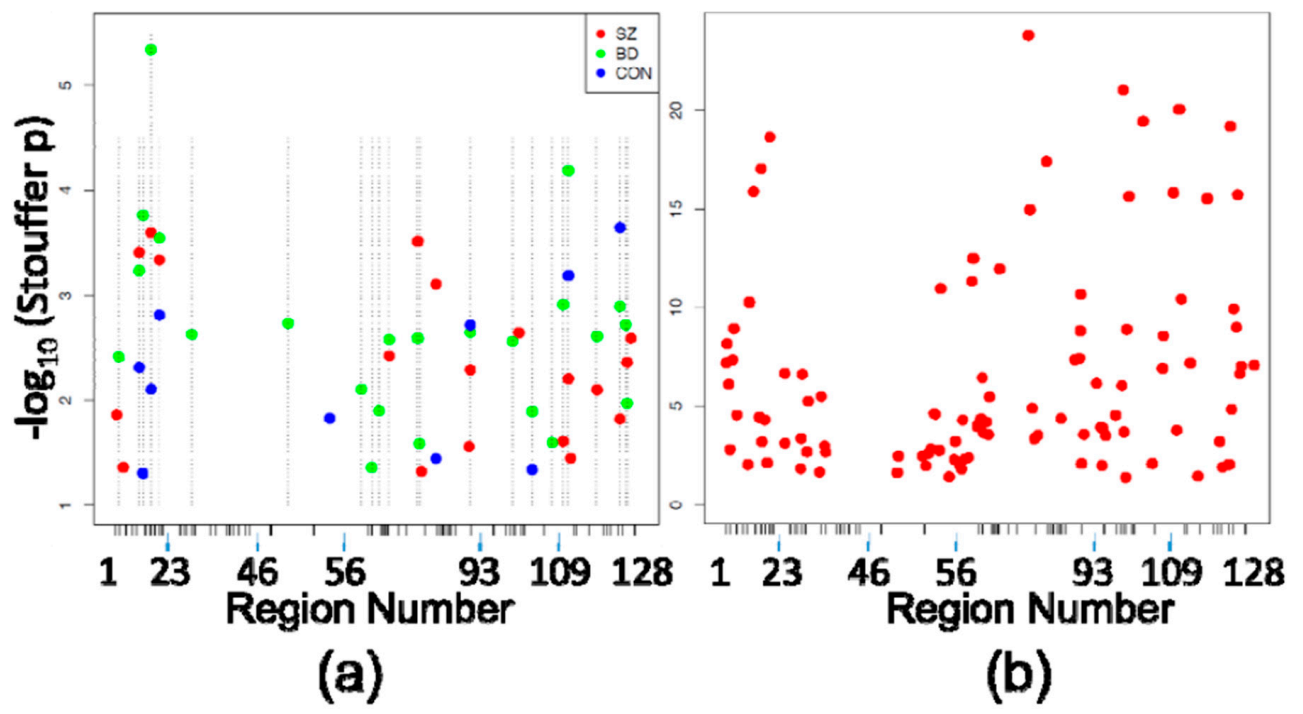

Figure 2. Differentially Methylated Regions (DMRegions). (a) Similar to Figure 1a, shown are the DMRegions identified within each eight-case diagnostic subgroup comparison (19 in SZ CA1 vs. SZ $\mathrm{CA} 2 / 3,23$ in BD CA1 vs. BD CA2/3, 10 in CON CA1 vs. CON CA2/3), with the $X$ axis again depicting the space corresponding to the $5476 \mathrm{HM} 450$ probes assessing sites within the 128 PGC regions, and the $\mathrm{Y}$ axis depicting the $-\log 10$ (Stouffer corrected $p$-value) of each DMRegion. X-axis ticks mark the boundaries between PGC regions. Dashed vertical lines indicate the locations of BD-associated DMRegions (the most abundant) to demonstrate the scarcity of DMRegions that are not shared across comparisons within the separate diagnostic subgroups; (b) The 103 DMRegions identified in comparison of all 24 CA1 samples vs. all 24 CA2/3 samples are plotted against the same axes as Figure 2a.

Table 3. Distribution and overlap of differentially methylated regions among circuit location comparisons.

\begin{tabular}{|c|c|c|c|c|c|c|c|}
\hline Chr & Start & End & Description & SZ & BD & $\mathrm{CON}$ & Pooled \\
\hline chr01 & 2381300 & 2381623 & $26 \mathrm{~kb}$ upstream of PLCH2 & 13 & & & 28 \\
\hline chr01 & 2391242 & 2391347 & $16 \mathrm{~kb}$ upstream of PLCH2 & & 16 & & \\
\hline chr01 & 8484005 & 8484417 & RERE intron 12 & 18 & & & 24 \\
\hline chr01 & 150123038 & 150123734 & PLEKH01 spans exon 2 & 3 & 5 & 5 & 21 \\
\hline chr01 & 150229144 & 150230345 & CA14 TSS & & 3 & 10 & 9 \\
\hline chr01 & 243584669 & 243584861 & SDCCAG8 intron \#15 & 1 & 1 & 6 & 8 \\
\hline chr02 & 72357938 & 72358024 & CYP26B1 3' end & 4 & 4 & 3 & 6 \\
\hline chr03 & 2553086 & 2553187 & CNTN4 intron 3 & & 11 & & 39 \\
\hline chr06 & 28499677 & 28499825 & GPX5 exon $33^{\prime}$ boundary & & 8 & & 83 \\
\hline chr07 & 1983170 & 1983503 & MADL1 intron 16 & & & 7 & 18 \\
\hline chr07 & 2143508 & 2144767 & MADL1 intron 12 & & 17 & & 17 \\
\hline chr07 & 86413439 & 86414302 & GRM3 intron 2 & & 23 & & 40 \\
\hline chr07 & 104909431 & 104909815 & SRPK2 exon $35^{\prime}$ boundary & & 19 & & 45 \\
\hline chr10 & 18689036 & 18689503 & CACNB2 exon $55^{\prime}$ boundary & 8 & 14 & & 16 \\
\hline chr11 & 46365894 & 46367100 & DGKZ exon 1 & 2 & 13 & & 1 \\
\hline chr11 & 46383032 & 46383209 & DGKZ exon 4 & & 22 & & 14 \\
\hline chr11 & 46401423 & 46401447 & DGKZ exon 32 & 19 & & & 47 \\
\hline chr11 & 57414402 & 57414908 & YPEL4 spans exon 2 & 5 & & 8 & 7 \\
\hline chr12 & 57589254 & 57589740 & LRP1 exon 53 & 16 & & & 29 \\
\hline chr12 & 57597137 & 57597238 & LRP1 exon $705^{\prime}$ boundary & 10 & 10 & 4 & 19 \\
\hline
\end{tabular}


Table 3. Cont.

\begin{tabular}{|c|c|c|c|c|c|c|c|}
\hline $\mathrm{Chr}$ & Start & End & Description & SZ & BD & CON & Pooled \\
\hline chr14 & 104171260 & 104172224 & XRCC3 intron 6 & & 15 & & 2 \\
\hline chr15 & 40599681 & 40600635 & PLCB2 TSS & 6 & & & 12 \\
\hline chr15 & 91426668 & 91427884 & Overlaps FURIN $3^{\prime}$ end \& FES TSS & & 20 & 9 & 4 \\
\hline chr16 & 30124804 & 30124904 & GDPD3 $3^{\prime}$ end & & 21 & & 36 \\
\hline chr16 & 67918485 & 67919362 & NRN1L TSS & 15 & 6 & & 10 \\
\hline chr16 & 67977866 & 67978450 & SLC12A4 exon 24 & 11 & 2 & 2 & 3 \\
\hline $\operatorname{chr} 16$ & 68000764 & 68001415 & CLC12A4 exon $25^{\prime}$ boundary & 17 & & & 20 \\
\hline chr17 & 18011514 & 18012134 & MYO15A TSS & 12 & 12 & & 13 \\
\hline chr20 & 37464180 & 37464594 & PPP1R16B exon 2 & 14 & 7 & 1 & 5 \\
\hline $\operatorname{chr} 22$ & 41613693 & 41613790 & L3MBTL2 intron 5 & & 9 & & 23 \\
\hline chr22 & 41636942 & 41637617 & CHADL TSS & 9 & 18 & & 11 \\
\hline $\operatorname{chr} 22$ & 42347907 & 42348061 & LINC00634 TSS & 7 & & & 35 \\
\hline
\end{tabular}

Listed are the locations of all DMRegions identified in the three within-diagnosis comparisons of CA1 and CA2/3. Numbers depict the rank order of that DMRegion in the indicated comparison, and missing numbers indicate that the associated region was not detected as significantly differentially methylated in that comparison.

\section{Discussion}

As our understanding of the complex structure of genetic risk for schizophrenia advances, an important next step in understanding this disease will be the difficult task of mapping that structure onto the complex neural circuitry of the human brain. Even in unicellular organisms, the relation between genotype and phenotype is highly complicated, as a given mutation informs phenotype through a set of modifiers within the genetic background that are specific to an individual organism $[17,18]$. Considering this single-cell complexity in concert with the extraordinary diversity of neuronal and glial cell types and circuits within the human brain, a robust understanding of how a genotype is elaborated into the unique phenotypes within the many cells that comprise the complex circuits found within individual cortical layers, regions, and subregions presents a formidable challenge both in health and in disease states such as schizophrenia.

Within the 108 PGC schizophrenia-associated genomic regions, we observed only a single DMPosition in all four of the diagnostic comparisons, as opposed to 497 DMPositions in the three circuit location-based comparisons. Similarly, there were no DMRegions identified in the four diagnostic comparisons, and a total of 52 DMRegions across all three circuit location comparisons. This finding of greater differences in DNA methylation patterns between brain regions from the same individual than exist within a single brain region across separate subjects is in keeping with previous work [8], and to our knowledge these data represent the most subtle comparison yet made. Interestingly, a more recent study [19] drew the exact opposite conclusion, finding that methylation differs more between individuals than between brain regions (with the exception of cerebellum compared to cerebral cortical regions). While the cause of this disagreement is unknown, multiple technical differences between the studies (Methylated DNA Immunoprecipitation Sequencing vs. MBD Affinity Purification Sequencing, distinct brain regions sampled, and different analytic techniques) may have contributed.

These data are consistent with another recently published study that investigated schizophrenia-associated DNA methylation changes across four distinct regions of the human brain in homogenized whole cortex, accounting for estimates of neuronal/glial proportions in each sample [20]. The prior study found multiple DMPositions and DMRegions between schizophrenia and control subjects, and while many were shared across brain regions, the majority of these changes were significant in only a subset of regions, with the cerebellum being the most distinct region assayed. In alignment with our findings, the prior study found no enrichment of schizophrenia-associated DMPositions or DMRegions within the 108 PGC regions. Very interestingly, this prior work found the association of DNA methylation with a schizophrenia polygenic risk score based on the PGC GWAS results to be much more consistent across brain regions than was the association of DNA methylation with a formal diagnosis of schizophrenia. 
As detailed in Table 2, the average methylation change for all DMPs observed in our analysis was $4.5 \%$, a value in keeping with many methylation change findings published from similar investigations $[12,20,21]$. The potential functional impact of a change of this magnitude within a complex heterogenous system such as the human hippocampus is difficult to predict and likely dependent on a great many factors, including the specific genomic context of each cytosine residue in question. While our laser-microdissected samples contain a more homogenous population of cells than samples of homogenized brain structures, they are still composed of multiple distinct cell types (i.e., glia and multiple GABAergic interneuronal cell types). Methylation changes are certainly not evenly distributed among all cells assessed by our measurements, and as such some populations are likely to host greater methylation differences between circuit locations and some populations lesser differences. As technologies continue to allow for the assessment of increasingly homogenous neuronal populations, effect sizes of identified disease and circuit location-associated methylation changes will likely increase. Another point in consideration of the functional importance of these differences is that the field is currently elucidating multiple roles for DNA methylation beyond the direct regulation of gene expression, including the regulation of alternate promoter usage, alternative splicing events, and support of transcriptional elongation [22].

While the samples employed here are far more specific than homogenized whole cortex, which is the most common method used to investigate human brain tissue, it is still essential to account for possible shifts in cellular populations between samples. The stratum oriens of the hippocampus contains exclusively GABAergic interneurons in addition to glial cells [14,23]. There exist tools to estimate the proportion of neuronal and non-neuronal cells within a sample based on its DNA methylation profile (i.e., the estimateCellCounts function within the minfi Bioconductor package [15]), however, these tools require a preexisting DNA methylation dataset from fluorescence-activated cell sorted (FACS) tissue. No such dataset is available for stratum oriens tissue from the human hippocampus, and available datasets from FACS sorted homogenized whole cortex are not applicable to our data. Previous work in our lab assessing gene expression profiles in equivalently microdissected stratum oriens tissue from CA1 and CA3 in a non-overlapping cohort of postmortem human hippocampus [24] found no significant difference between circuit locations in the expression of the glial specific mRNA marker GFAP (encoding glial fibrilary acidic protein) or the neuron specific mRNA ENO2 (encoding enolase 2, also known as neuron specific enolase) as normalized to the expression of the housekeeping gene GAPDH (encoding glyceraldehyde 3-phosphate dehydrogenase). Based on these findings, we are confident that the reported DNA methylation changes between the stratum oriens of CA1 and CA3 are not due to different proportions of neurons and glia present at these circuit locations, but instead depict distinct methylation profiles within phenotypically equivalent cellular populations.

The present analyses included a relatively small cohort, and greater numbers may have identified additional DMPositions or DMRegions, but the general observation of more between circuit location differences than between-subject differences is still valid. Additionally, the lack of genotyping data for the subjects precluded searching for meQTLs within the PGC regions in our cohort, as others have done previously $[11,12]$. One further potential limitation of this study is our inability to investigate CpG sites within the PGC regions not targeted by probes on the HM450 BeadArray. Indeed, there are 25 PGC regions associated with zero HM450 probes, and another 14 regions associated with only a single probe. Again, these shortcomings do not undermine conclusions regarding the within vs. between-subject comparisons described above.

While patients within the SZ and BD groups were treated with psychotropic medications [13], we argue that medication is not driving the observed changes in DNA methylation for two reasons. First, we observed more DNA methylation differences within between-circuit comparisons, where medication exposure is perfectly balanced as the same individuals are present in the CA1 group and the CA2/3 group, as opposed to comparisons across diagnostic groups of separate individuals. Second, there is a large degree of overlap in DMPositions and DMRegions found in the three diagnostic 
groups, including the non-medication exposed control group. We cannot exclude the possibility that medication exposure is causal of the twofold greater numbers of DMPositions and DMRegions observed in the $\mathrm{SZ}$ and $\mathrm{BD}$ groups as compared to the $\mathrm{CON}$ group, and this greater difference across circuit locations in psychotic disorders as a result of either the disease process itself or its pharmacologic treatment is an interesting finding requiring further study.

\section{Conclusions}

Even as basic a molecular phenotype as DNA methylation patterns shows variation across closely related cellular populations such as those found within a single cellular layer in distinct subfields of the human hippocampus within individual subjects. The 108 PGC schizophrenia-associated genomic regions represent a subset of the genome where inter-individual genetic variation is likely to be especially relevant to an individual's risk for schizophrenia. Our finding of greater differences in DNA methylation of these regions between circuit locations within the identical genetic background of the same individuals as compared to the differences between genetically diverse groups of patients and controls illustrates the difficulties of determining the impact of a given genotype on the complex phenotypes encompassed by a neuropsychiatric illness such as schizophrenia. Understanding the influence of genetic variants on the complex landscape of schizophrenia risk within the many distinct cell types, circuits, tissue microenvironments, and time points relevant to this disease is the next necessary step in this ongoing and grand challenge.

Supplementary Materials: The following are available online at www.mdpi.com/2073-4425/8/5/143/s1.

Acknowledgments: This work was supported by grants MH077175 (Benes) and MH/NS077550 (Benes). W. Brad Ruzicka was supported in part by the Andrew P. Merrill Memorial Research Fellowship and the Maria Lorenz Pope Fellowship from McLean Hospital, a NARSAD Young Investigator Award from the Brain and Behavior Research Foundation, the MD/PhD Fellowship from the American Psychiatric Association and Pfizer Pharmaceuticals, and a Dupont-Warren Fellowship from Harvard Medical School.

Author Contributions: W. Brad Ruzicka and Francine M. Benes conceived and designed the experiments; W. Brad Ruzicka and Sivan Subburaju performed the experiments; W. Brad Ruzicka analyzed the data; W. Brad Ruzicka wrote the paper.

Conflicts of Interest: The authors declare no conflict of interest.

\section{References}

1. Schizophrenia Working Group of the Psychiatric Genomics Consortium. Biological insights from 108 schizophrenia-associated genetic loci. Nature 2014, 511, 421-427.

2. Ng, S.B.; Turner, E.H.; Robertson, P.D.; Flygare, S.D.; Bigham, A.W.; Lee, C.; Shaffer, T.; Wong, M.; Bhattacharjee, A.; Eichler, E.E.; et al. Targeted capture and massively parallel sequencing of 12 human exomes. Nature 2009, 461, 272-276. [CrossRef] [PubMed]

3. Cnv and Schizophrenia Working Groups of the Psychiatric Genomics Consortium. Contribution of copy number variants to schizophrenia from a genome-wide study of 41,321 subjects. Nat. Genet. 2017, 49, $27-35$.

4. Fatemi, S.H.; Folsom, T.D. The neurodevelopmental hypothesis of schizophrenia, revisited. Schizophr. Bull. 2009, 35, 528-548. [CrossRef] [PubMed]

5. McCarty, R. Cross-fostering: Elucidating the effects of genexenvironment interactions on phenotypic development. Neurosci. Biobehav. Rev. 2017, 73, 219-254. [CrossRef] [PubMed]

6. Raj, A.; Rifkin, S.A.; Andersen, E.; van Oudenaarden, A. Variability in gene expression underlies incomplete penetrance. Nature 2010, 463, 913-918. [CrossRef] [PubMed]

7. Schmitt, A.; Martins-de-Souza, D.; Akbarian, S.; Cassoli, J.S.; Ehrenreich, H.; Fischer, A.; Fonteh, A.; Gattaz, W.F.; Gawlik, M.; Gerlach, M.; et al. Consensus paper of the WFSBP Task Force on Biological Markers: Criteria for biomarkers and endophenotypes of schizophrenia, part III: Molecular mechanisms. World J. Biol. Psychiatry 2016. [CrossRef] [PubMed]

8. Davies, M.N.; Volta, M.; Pidsley, R.; Lunnon, K.; Dixit, A.; Lovestone, S.; Coarfa, C.; Harris, R.A.; Milosavljevic, A.; Troakes, C.; et al. Functional annotation of the human brain methylome identifies tissue-specific epigenetic variation across brain and blood. Genome Biol. 2012. [CrossRef] [PubMed] 
9. Hannon, E.; Lunnon, K.; Schalkwyk, L.; Mill, J. Interindividual methylomic variation across blood, cortex, and cerebellum: Implications for epigenetic studies of neurological and neuropsychiatric phenotypes. Epigenetics 2015, 10, 1024-1032. [CrossRef] [PubMed]

10. Hoffmann, A.; Ziller, M.; Spengler, D. The Future is The Past: Methylation QTLs in Schizophrenia. Genes 2016, 7, 104. [CrossRef] [PubMed]

11. Jaffe, A.E.; Gao, Y.; Deep-Soboslay, A.; Tao, R.; Hyde, T.M.; Weinberger, D.R.; Kleinman, J.E. Mapping DNA methylation across development, genotype and schizophrenia in the human frontal cortex. Nat. Neurosci. 2016, 19, 40-47. [CrossRef] [PubMed]

12. Hannon, E.; Spiers, H.; Viana, J.; Pidsley, R.; Burrage, J.; Murphy, T.M.; Troakes, C.; Turecki, G.; O'Donovan, M.C.; Schalkwyk, L.C.; et al. Methylation QTLs in the developing brain and their enrichment in schizophrenia risk loci. Nat. Neurosci. 2016, 19, 48-54. [CrossRef] [PubMed]

13. Ruzicka, W.B.; Subburaju, S.; Benes, F.M. Circuit- and Diagnosis-Specific DNA Methylation Changes at gamma-Aminobutyric Acid-Related Genes in Postmortem Human Hippocampus in Schizophrenia and Bipolar Disorder. JAMA Psychiatry 2015, 72, 541-551. [CrossRef] [PubMed]

14. Freund, T.F.; Buzsaki, G. Interneurons of the hippocampus. Hippocampus 1996, 6, 347-470. [CrossRef]

15. Aryee, M.J.; Jaffe, A.E.; Corrada-Bravo, H.; Ladd-Acosta, C.; Feinberg, A.P.; Hansen, K.D.; Irizarry, R.A. Minfi: A flexible and comprehensive Bioconductor package for the analysis of Infinium DNA methylation microarrays. Bioinformatics 2014, 30, 1363-1369. [CrossRef] [PubMed]

16. Peters, T.J.; Buckley, M.J.; Statham, A.L.; Pidsley, R.; Samaras, K.; Lord, R.V.; Clark, S.J.; Molloy, P.L. De novo identification of differentially methylated regions in the human genome. Epigenet. Chromatin. 2015. [CrossRef]

17. Dowell, R.D.; Ryan, O.; Jansen, A.; Cheung, D.; Agarwala, S.; Danford, T.; Bernstein, D.A.; Rolfe, P.A.; Heisler, L.E.; Chin, B.; et al. Genotype to phenotype: A complex problem. Science 2010, 328, 469. [CrossRef] [PubMed]

18. Linder, R.A.; Seidl, F.; Ha, K.; Ehrenreich, I.M. The complex genetic and molecular basis of a model quantitative trait. Mol. Biol. Cell 2016, 27, 209-218. [CrossRef] [PubMed]

19. Illingworth, R.S.; Gruenewald-Schneider, U.; De Sousa, D.; Webb, S.; Merusi, C.; Kerr, A.R.; James, K.D.; Smith, C.; Walker, R.; Andrews, R.; et al. Inter-individual variability contrasts with regional homogeneity in the human brain DNA methylome. Nucleic Acids Res. 2015, 43, 732-744. [CrossRef] [PubMed]

20. Viana, J.; Hannon, E.; Dempster, E.; Pidsley, R.; Macdonald, R.; Knox, O.; Spiers, H.; Troakes, C.; Al-Saraj, S.; Turecki, G.; et al. Schizophrenia-associated methylomic variation: Molecular signatures of disease and polygenic risk burden across multiple brain regions. Hum. Mol. Genet. 2017, 26, 210-225. [CrossRef] [PubMed]

21. Gibbs, J.R.; van der Brug, M.P.; Hernandez, D.G.; Traynor, B.J.; Nalls, M.A.; Lai, S.-L.; Arepalli, S.; Dillman, A.; Rafferty, L.P.; Troncoso, J.; et al. Abundant quantitative trait loci exist for DNA methylation and gene expression in human brain. PLoS Genet. 2010, 6, e1000952. [CrossRef] [PubMed]

22. Jones, P.A. Functions of DNA methylation: Islands, start sites, gene bodies and beyond. Nat. Rev. Genet. 2012, 13, 484-492. [CrossRef] [PubMed]

23. Benes, F.M.; Berretta, S. GABAergic interneurons: Implications for understanding schizophrenia and bipolar disorder. Neuropsychopharmacology 2001, 25, 1-27. [CrossRef]

24. Benes, F.M.; Lim, B.; Matzilevich, D.; Walsh, J.P.; Subburaju, S.; Minns, M. Regulation of the GABA cell phenotype in hippocampus of schizophrenics and bipolars. Proc. Natl. Acad. Sci. USA 2007, 104, 10164-10169. [CrossRef] [PubMed]

(C) 2017 by the authors. Licensee MDPI, Basel, Switzerland. This article is an open access article distributed under the terms and conditions of the Creative Commons Attribution (CC BY) license (http://creativecommons.org/licenses/by/4.0/). 